\title{
Cetoacidosis diabética severa secundaria a síndrome de resistencia a la insulina tipo $B$
}

\author{
Leopoldo J. Rodríguez-Cruz*, Gerardo Capiz-Lemus y Roberto de J. Sandoval-Muñiz \\ Departamento de Urgencias Médicas, Servicios Médicos Municipales de Guadalajara Cruz Verde, Guadalajara, Jalisco, México
}

\section{Resumen}

Introducción: El síndrome de resistencia a la insulina tipo B (SRI-B) es un desorden autoinmune ocasionado por la biosíntesis de anticuerpos policlonales dirigidos contra receptores especificos de insulina. Estos anticuerpos poseen un efecto insulinomimético, es decir, al aumentar su concentración sérica se incrementa la degradación del receptor de insulina y provoca la resistencia a la insulina. Objetivo: Presentar el caso clínico y revisión de literatura sobre paciente con cetoacidosis diabética grave como complicación de SRI-B en el área de urgencias. Materiales y métodos: Se presenta caso clínico de paciente de sexo femenino de 40 años de edad, quien inicia en forma súbita su padecimiento, con diagnóstico previo de diabetes mellitus tipo 2, hipotiroidismo e hipertensión arterial sistémica, y debido a los altos requerimientos de insulina se investigó síndrome de resistencia a la insulina. Conclusiones: El uso de esteroides de manera temprana en pacientes con sospecha de resistencia a insulina mejora la supervivencia del paciente y acortan la estancia en la sala de urgencias o terapia intensiva.

Palabras clave: SRI-B. Síndrome de resistencia a la insulina tipo B. Corticosteroides. Cetoacidosis. Inmunosupresores.

\section{Severe diabetic ketoacidosis secondary to type B insulin resistance syndrome}

\begin{abstract}
Introduction: Type B Insulin Resistance Syndrome (SRI-B), is an autoimmune disorder caused by the biosynthesis of polyclonal antibodies directed against specific insulin receptors. These antibodies have a mimetic effect of insulin; that is, by increasing its serum concentration, the degradation of the insulin receptor increases and causes insulin resistance. Objective: The objective of this report is to present a clinical case and literature review of a patient who suffered severe diabetic ketoacidosis (CAD) and type $B$ insulin resistance syndrome as a medical emergency. Materials and methods: $A$ clinical case is presented of a 40-year-old female patient, who suddenly started her condition, with a previous diagnosis of Type 2 Diabetes Mellitus (DM2), hypothyroidism, systemic arterial hypertension (HAS), and due to the high insulin requirements insulin resistance syndrome was investigated. Conclusions: The use of steroids early in patients with suspected insulin resistance improves patient survival and shortens the stay in the emergency room or intensive care.
\end{abstract}

Keywords: SRI-B. Corticosteroids. Ketoacidosis. Immunosuppressants.

\section{Correspondencia:}

*Leopoldo J. Rodríguez-Cruz

E-mail: polo_mixtavda@ hotmail.com
Disponible en internet: 10-11-2021 Rev Educ Investig Emer. 2022;4(1):45-49 www.medicinadeemergencias.com

2604-6520 @ 2021 Sociedad Mexicana de Medicina de Emergencias, AC. Publicado por Permanyer México SA de CV. Este es un artículo open access bajo la licencia CC BY-NC-ND (http://creativecommons.org/licenses/by-nc-nd/4.0/). 


\section{Introducción}

El síndrome de resistencia a la insulina tipo B (SRI-B) es un desorden autoinmune poco común que se produce por la biosíntesis de anticuerpos policlonales dirigidos contra epítopos específicos del receptor de insulina. Esta patología se presenta principalmente en mujeres afroamericanas de 30 a 50 años de edad y tiene una elevada tasa de mortalidad ${ }^{1}$. La prevalencia exacta de esta enfermedad es desconocida y no existen estudios poblacionales que aporten información precisa al respecto? ${ }^{2}$.

Los autoanticuerpos altamente específicos hacia el receptor de insulina tienen un efecto insulinomimético, es decir, al aumentar su concentración sérica se produce un incremento en la degradación del receptor de insulina ubicado en la superficie celular, ocasionando resistencia a esta hormona ${ }^{3}$.

Estos cambios moleculares previamente mencionados se observan de manera clínica en un paciente con SRI-B por la presencia de acantosis nigricans, ovario poliquístico e hiperandrogenismo, aunque estos signos no son patognomónicos de esta patología 4 .

En cuanto a los hallazgos inmunológicos, la mayor parte de los pacientes que desarrollan SRI-B también manifiestan frecuentemente comorbilidades asociadas como lupus eritematoso sistémico ${ }^{5,6}$.

Por otro lado, como consecuencia del SRI-B se presentan cambios metabólicos y bioquímicos como la hiperglucemia no cetósica, que puede ser seguida por hipoglucemia reactiva o en ayunas. Raramente se puede observar hipoglucemia aislada ${ }^{7}$. Estos hallazgos metabólicos más frecuentes como la hiperglucemia e hipoglucemia son la principal causa de mortalidad en estos pacientes, con reportes de hasta un $50 \%$ de los casos. Ambas condiciones no son excluyentes, y se puede pasar de una fase de hiperglucemia a una de hipoglucemia bruscamente, lo cual es un dato de mal pronóstico en los pacientes ${ }^{8}$.

Lo anterior se puede explicar por la disminución de la concentración sérica de autoanticuerpos, que favorecen la acción insulinomimética, menor degradación de receptores de insulina y la consecuente disminución de la concentración sérica de glucosa en el paciente, debida a la mayor translocación de glucosa al interior de la célula9,10.

Estas diferentes manifestaciones de la interacción entre receptor y los anticuerpos pueden explicarse ampliamente debido a las diferencias cuantitativas en los títulos de anticuerpos ${ }^{11}$. A pesar de la etiología y patogenia definida de este trastorno, la terapéutica empleada actualmente no es satisfactoria, solo sintomática para los estados metabólicos que de este trastorno se derivan ${ }^{12,13}$.

\section{Fisiopatología}

El SRI- B es causado por anticuerpos policlonales inmunoglobulina $(\mathrm{Ig}) \mathrm{G}$ que van dirigidos contra el receptor de insulina, lo cual afecta la homeostasis de la glucosa ${ }^{14}$. La formación de autoanticuerpos es una de las características de las enfermedades autoinmunes y el lupus eritematoso sistémico es la enfermedad más común que se presenta como complicación de este síndrome ${ }^{15}$.

\section{Presentación del caso}

Mujer de 40 años de edad, con obesidad grado III, diabetes mellitus 2 con mal apego al tratamiento, aplicación de insulina glargina e insulina rápida 30 UI vía subcutánea (SC) todos los días; hipertensión arterial sistémica e hipotiroidismo sin tratamiento (Fig. 2). Inicia su padecimiento con náuseas y vómitos, se realiza glucemia capilar en su domicilio, obteniéndose el resultado de «error» por estar fuera de los rangos detectables, motivo por el cual se administra insulina de acción rápida vía SC, sin mejoría, exacerbándose el cuadro y presentando alteración del estado de alerta y diaforesis.

Por lo anterior, la paciente es trasladada a la unidad Cruz Verde Ruiz Sánchez, donde es atendida. Inicialmente se determina una glucosa sérica central de $800 \mathrm{mg} / \mathrm{dl}$, se solicitan estudios de laboratorio como una gasometría arterial, con resultado de acidosis metabólica severa. Posteriormente, se inicia manejo intravenoso con solución salina al $0.9 \%$, de igual forma, se realiza determinación de potasio sérico para iniciar con infusión de insulina rápida $(0.14 \mathrm{Ul} / \mathrm{kg} / \mathrm{hora})$. En la evaluación neurológica se documenta deterioro del estado neurológico mayor a 2 puntos del basal respecto de su ingreso, por escala de coma de Glasgow, por lo que se decide abordar de manera avanzada la vía aérea para protegerla y se inicia ventilación mecánica asistida. Durante el tratamiento previamente mencionado, la paciente comienza con deterioro hemodinámico requiriendo soporte de aminas vasoactivas (norepinefrina $0.1 \mathrm{mcg} / \mathrm{kg} / \mathrm{h}$ ) con pobre respuesta al manejo inicial, evidenciando choque refractario, por lo que se decide agregar un segundo vasopresor a la terapéutica empleada hasta el momento. La hiperglucemia y acidosis metabólica persisten en la paciente; aunado a esto, se 


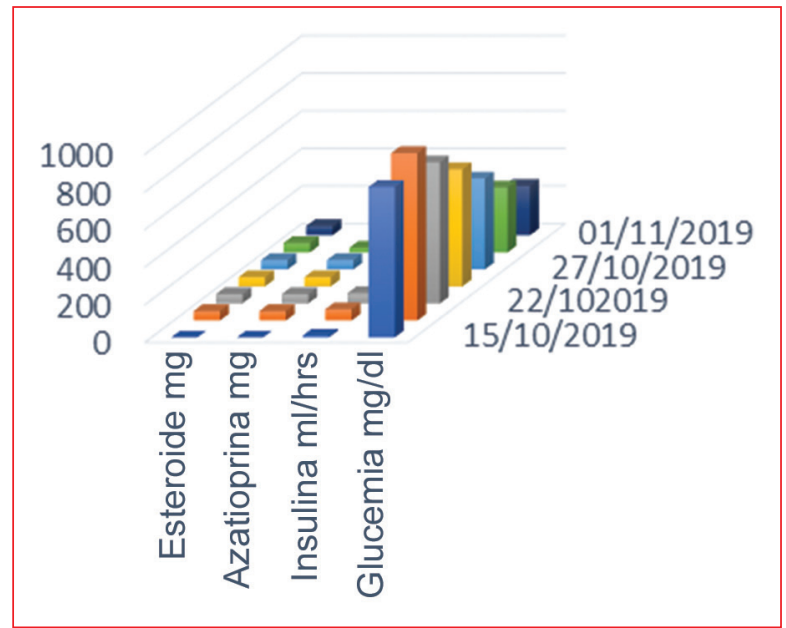

Figura 1. Grafico de comparación de terapéutica utilizada en la paciente y disminución de glucosa sérica, con cada uno de los tratamientos empleados y de insulina intravenosa $(\mathrm{ml} / \mathrm{hr})$.

obtiene osmolaridad sérica, la cual resulta elevada (305 Osm) y se procede traslado a la unidad de terapia intensiva (UTI) en tercer nivel, en donde a su ingreso se interconsulta a endocrinología por los altos requerimientos de insulina (mayor a 2,800 UI/día), quienes sugieren resistencia a la insulina por la presencia de anticuerpos contra insulina. Se solicita determinación de anticuerpos contra el receptor de insulina y se demuestra una concentración sérica «positiva» con títulos de $100 \mathrm{ul} / \mathrm{ml}$ contra insulina.

Permaneció en el servicio de UTI durante 15 días con la siguiente evolución:

- Neurológico: bajo sedación y analgesia con midazolam y buprenorfina, para mantener RASS (Richmond Agitation-Sedation Scale) 4 y Ramsay 5.

- Cardiovascular: a su ingreso con uso de aminas, norepinefrina por hipotensión arterial, llenado capilar retardado, choque hipovolémico.

- Respiratorio: fase III de ventilación, modo A/C volumen, presión positiva al final de la espiración 5 $\mathrm{cmH}_{2} \mathrm{O}$, fracción inspiratoria de oxígeno $60 \%$, frecuencia respiratoria $14 \mathrm{rpm}$, campos pulmonares sin crépitos o sibilancias, sin ruidos agregados, lo cual permitió procedimiento de destete (weaning) exitoso, extubándose al 5. día, sin complicaciones.

- Hídrico y renal: presenta lesión renal aguda KDIGO (Kidney Disease: Improving Global Outcomes) 1, incremento de creatinina sérica $3.2 \mathrm{mg} / \mathrm{dl}$, urea sérica $126 \mathrm{mg} / \mathrm{dl}$, con volumen de líquidos en las primeras 12 horas de 7 litros por estado mixto, con mejoría progresiva hasta su egreso.

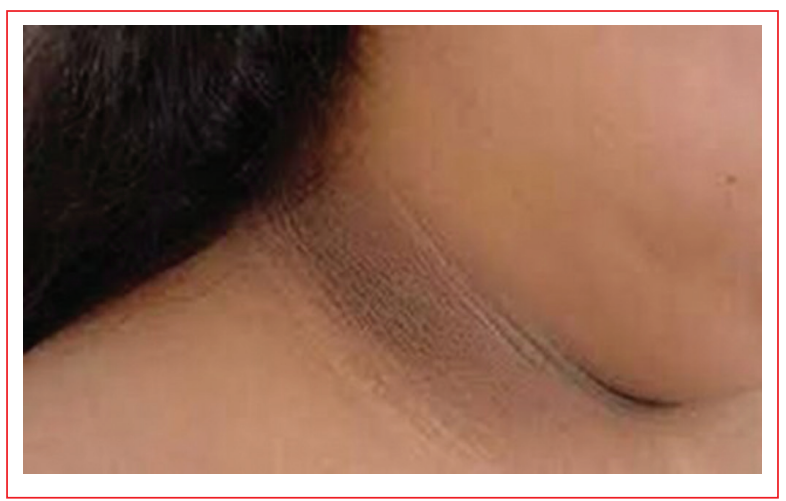

Figura 2. Mujer de 40 años de edad, que muestra acantosis nigricans (respetado el derecho a la privacidad y con consentimiento informado por parte de la paciente).

- Gastrometabólico: en ayuno, glucosa sérica $743 \mathrm{mg} / \mathrm{dl}$, persistente con glucosa capilar $500 \mathrm{mg} / \mathrm{dl}$ con uso de esteroide y azatioprina, logrando regulación metabólica de glucosa sérica a $126 \mathrm{mg} / \mathrm{dl}$.

- Hematoinfeccioso: febril intermitente, leucocitosis con predominio de neutrófilos, con evidencia de foco infeccioso de origen urinario, uso de levofloxacino de forma empírica, solicitándose cultivos, sin datos de sangrado o coagulopatía y lactato sérico $1.6 \mathrm{mmol} / \mathrm{l}$. Se decide manejo con bolos de esteroide (metilprednisolona), posteriormente se cambia a terapia con prednisona a dosis de $50 \mathrm{mg}$ cada 24 horas en combinación con inmunosupresor, azatioprina $50 \mathrm{mg}$ cada 12 horas(Fig. 1), con lo cual la paciente presenta rápida mejoría del estado clínico y metabólico, así como reducción de las necesidades de insulina hasta $3 \mathrm{UI} / \mathrm{kg}$ (Fig. 3).

\section{Discusión}

Hong, et al. (2018) describieron tres tipos de resistencia a la insulina: a) tipo $A$, caracterizado por defecto estructural en el receptor de la insulina; b) tipo B, existe síntesis de autoanticuerpos contra el receptor de la insulina, y c) tipo C, asociado a un deterioro del receptor de tirosina cinasa ${ }^{4}$. Actualmente se reportan terapias alternativas que incluyen plasmaféresis, inmunoglobulina intravenosa y dosis altas de esteroides.

El objetivo de nuestro trabajo es realizar una revisión en la literatura sobre el tema y reportar el caso de un paciente con diagnóstico de cetoacidosis diabética severa secundaria a un SRI-B manejado en el servicio de urgencias durante 5 días (Figs. 1 y 2), para 
Tabla 1. Tabla de comparación de terapéutica utilizada en la paciente y disminución de glucosa sérica, con cada uno de los tratamientos empleados y de insulina intravenosa $(\mathrm{ml} / \mathrm{h})$

\begin{tabular}{|l|l|l|l|l|}
\hline Fecha & Esteroide & Azatioprina & Insulina & Glucemia \\
\hline $15 / 10 / 19$ & & & $0.14 \mathrm{ui} / \mathrm{kg} / \mathrm{hra}$ & $743 \mathrm{mg} / \mathrm{dl}$ \\
\hline $22 / 10 / 19$ & & $0.8 \mathrm{ui} / \mathrm{kg} / \mathrm{hra}$ & $800 \mathrm{mg} / \mathrm{dl}$ \\
\hline $27 / 10 / 19$ & & & $1.16 \mathrm{ui} / \mathrm{kg} / \mathrm{hra}$ & $500 \mathrm{mg} / \mathrm{dl}$ \\
\hline $01 / 11 / 19$ & $50 \mathrm{mg} / \mathrm{dia}$ & & $0.05 \mathrm{ui} / \mathrm{kg} / \mathrm{hra}$ & $126 \mathrm{mg} / \mathrm{dl}$ \\
\hline
\end{tabular}

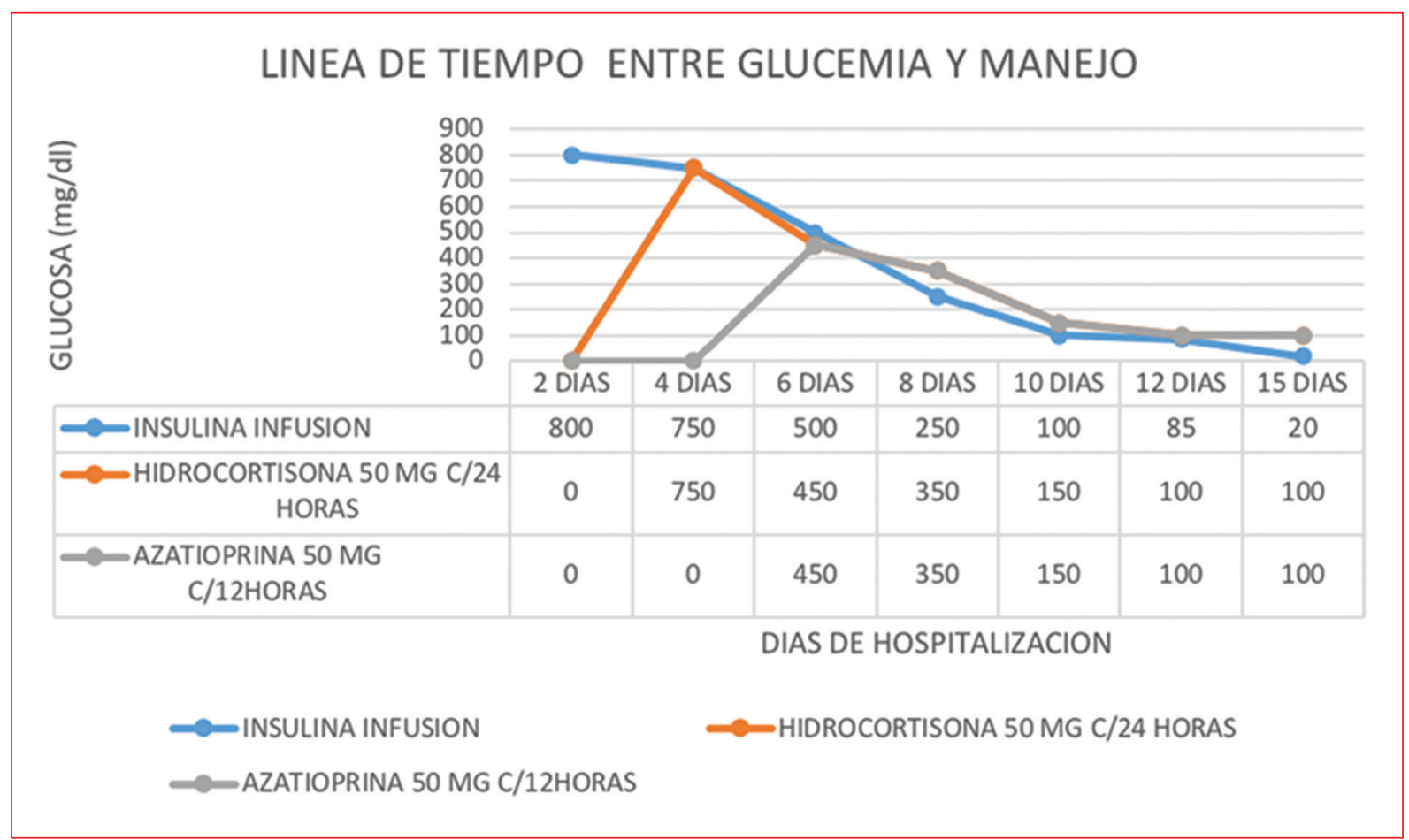

Figura 3. Línea de tiempo entre los niveles de glucosa sérica y manejo establecido con hidrocortisona, azatioprina y la infusión de insulina rápida.

posteriormente ser derivada a hospital de tercer nivel de atención para manejo multidisciplinario.

En este caso se trata de una paciente diagnosticada con SRI-B con presentación clínica atípica, sin embargo, requirió cantidades altas de insulina terapéutica, por lo que es importante realizar un diagnóstico oportuno e iniciar un manejo a base de corticosteroides en combinación con inmunosupresores, para poder llegar a las metas terapéuticas ya establecidas en múltiples ensayos y diversos artículos.

Esta evidencia es de suma importancia para los médicos de urgencias. ya que algunos casos de SRI-B se presentan de manera atípica, y la detección oportuna disminuye la morbimortalidad del paciente con la terapéutica indicada.

\section{Conclusión}

EI SRI- B es un trastorno de difícil diagnóstico y con baja incidencia, el cual tiene buen pronóstico y supervivencia de los pacientes, acortando sus días de estancia en la UTI con el uso de corticosteroides en combinación con inmunosupresores. No obstante, el abordaje diagnóstico puede ser difícil debido a la clínica atípica en estos pacientes, y la evidencia hasta el momento indica que el tratamiento anticipado conlleva 
recuperación y acorta los días de estancia hospitalaria.

\section{Agradecimientos}

Al personal de enfermería, administrativos, de laboratorio, y a mis médicos adscritos al servicio de urgencias que apoyaron para realizar este proyecto. A mis maestros por apoyarme, Dra. Claudia Santana, Dra. Sonia Carreola y Dr. Roberto Sandoval.

\section{Financiamiento}

Los autores no recibieron patrocinio para llevar a cabo este artículo.

\section{Conflicto de intereses}

Los autores declaran no tener conflicto de intereses alguno.

\section{Responsabilidades éticas}

Protección de personas y animales. Los autores declaran que para esta investigación no se han realizado experimentos en seres humanos ni en animales.

Confidencialidad de los datos. Los autores declaran que han seguido los protocolos de su centro de trabajo sobre la publicación de datos de pacientes.

Derecho a la privacidad y consentimiento informado. Los autores han obtenido el consentimiento informado de los pacientes y/o sujetos referidos en el artículo. Este documento obra en poder del autor de correspondencia.

\section{Bibliografía}

1. Gutiérrez RC, Roura GA. Mecanismos moleculares de la resistencia a la insulina; una actualización. Gac Med Mex. 2017;153:214-28.

2. Klubo-Gwiezdzinska J, Lange M, Cochran E, Semple RK, Gewert C Brown RJ, et al. Combined immunosuppressive therapy induces remission in patients with severe type $B$ insulin resistance: A prospective cohort study. Diabetes Care. 2018;41(11):2353-60.

3. Hwa HJ, Jin KH, Seo PK, Jeong KB. Paradigm shift in the management of type B insulin resistance. Ann Transl Med. 2018;6(2):S98.

4. Arioglu E, Andewelt A, Diabo C, Bell M, Taylor SI, Gorden P. Clinical course of the syndrome of autoantibodies to the insulin receptor (type $B$ insulin resistance): a 28-years perspective. Medicine (Baltimore). 2002;81(2):87-100.

5. Page KA, Dejardin S, Kahn CR, Kulkarni RN, Herold KC, Inzucchi SE. A patient with type $B$ insulin resistance syndrome, responsive to immune therapy. Nat Clin Pract Endocrinol Metab. 2007;3(12):835-40.

6. Malek R, Chong AY, Lupsa BC, Lungu AO, Cochran EK, Soos MA, et al. Treatment of type $B$ insulin resistance: a novel approach to reduce insulin receptor autoantibodies. J Clin Endocrinol Metab. 2010;95(8):3641-7.

7. Eriksson JW, Bremell T, Eliasson B, Fowelin J, Fredriksson L, Yu ZW. Successful treatment with plasmapheresis, cyclophosphamide, and cyclosporin A in type B syndrome of insulin resistance. Case report. Diabetes Care. 1998;21(8):1217-20.

8. Coll AP, Thomas S, Mufti GJ. Rituximab therapy for the type B syndrome of severe insulin resistance. N Engl J Med. 2004;350(3):310-1.

9. Lebkowska A, Krentowska A, Adamska A, Piasecka B, Lipinska D. Type $B$ insulin resistance syndrome in a patient with connective tissue disease. Endocrine Abstracts. 2017;49:EP466.

10. Mantzoros C. Insulin resistance: Definition and clinical spectrum [Internet]. UpToDate [acceso: 1 julio 2020]. Disponible en: https://www.uptodate.com

11. Khokher MA, Avasthy N, Taylor AM, Fonseca VA, Dandona P. Insulin-receptor antibody and hypoglycemia associated with Hodgkin's disease. Lancet. 1987;1(8534):693-4.

12. Blecher M. Receptors, antibodies and disease. Clin Chem. 1984;30(7):1137-56.

13. Nagayama $Y$, Morita H, Komukai D, Watanabe S, Yoshimura A. Type B insulin resistance syndrome induced by increased activity of systemic lupus erythematosus in a hemodialysis patient. Clin Nephrol. 2008:69(2):130-4.

14. Coll AP, Thomas S, Mufti GJ. Rituximab therapy for the type B syndrome of severe insulin resistance. N Engl J Med. 2004;350:310-1.

15. Tsokos GC, Gorden P, Antonovych T, Wilson CB, Balow JE. Lupus nephritis and other autoimmune features in patients with diabetes mellitus due to autoantibody to insulin receptors. Ann Intern Med. 1985;102(2): 176-81 